\title{
O aproveitamento do resíduo da indústria do sisal no controle de larvas de mosquitos
}

\author{
Utilization of the waste of sisal industry in the \\ control of mosquito larvae
}

\author{
Ana Paula B. Pizarro, Alfredo M. Oliveira filho, José P. Parente, Marli T.V. \\ Melo, Celso $\epsilon$. dos Sontos e Paulo R. Lima
}

\begin{abstract}
Resumo Descreve-se o aproveitamento do resíduo do desfibramento das folhas de Agave sisalana, como um larvicida para o combate a mosquitos transmissores de doenças tropicais. Durante 24 horas, larvas de Aedes aegypti e Culex quinquefasciatus foram expostas a concentrações diferentes do extrato da planta para determinar as concentrações letais. Para A. aegypti foi constatada a CL50 em 322ppm e para C. quinquefasciatus em 183ppm. Foi investigada a ação de saponinas existentes na planta, ficando evidenciado que o resíduo de A. sisalana é ativo através da interação de vários dos seus componentes. Este extrato poderá ser utilizado em campo, na concentração de 100ppm para C. quinquefasciatus com um aumento do tempo de exposição para três dias, obtendo-se uma mortalidade de $100 \%$ das larvas. Este produto, porém, não é recomendado para o controle de A. aegypti, devido à necessidade de uma alta concentração para a obtenção de $100 \%$ de mortalidade das larvas e ao fato destas se desenvolverem preferencialmente em água potável.
\end{abstract}

Palavras-chaves: Sisal. Agave sisalana. Aedes aegypti. Culex quinquefasciatus. Controle de vetores.

\begin{abstract}
The aim of this research was to utilize the waste residues of sisal fiber separation from Agave sisalana leaves to develop a larvicide for the combat of mosquito transmitting tropical diseases. Larvae of Aedes aegypti and Culex quinquefasciatus were exposed to different concentrations of the Agave extract for 24 hours to determine lethal concentrations. The LC50 for A. aegypti was $322 \mathrm{ppm}$ and the LC50 for C. quinquefasciatus was $183 \mathrm{ppm}$. To detect the active substances, saponins were investigated. It was found that the various components of the extract were effective in eliminating the larvae. Under field conditions, this formulation can probably be used at $100 \mathrm{ppm}$, wich causes 100\% mortality of C. quinquefasciatus larvae after 3-4 days. The product is not recommended for use against $\mathbf{A}$. aegypti due to the necessity for high concentrations and to the fact that the larvae of this species live frequently on drinking water. To avoid fermentation, Agave extract should be used in a dehydrated form which also represent a good formulation for practical use.
\end{abstract}

Key-words: Sisal. Agave sisalana. Aedes aegypti. Culex quinquefasciatus. Vector control.

\footnotetext{
Laboratórios de Biologia e de Química de Carboidratos do Núcleo de Pesquisas de Produtos Naturais (NPPN), Universidade Federal do Rio de Janeiro, Rio de Janeiro, RJ.

Coordenação dos Programas de Pós-Graduação em Engenharia (COPPE), Universidade Federal do Rio de Janeiro, RJ. Trabalho realizado com auxílio do CNPq (Bolsa n 141208/94-5), CEPEG-UFRJ, WHO/World Bank/TDR.

Endereço para correspondência: Dr. Alfredo Martins de Oliveira Filho. Laboratório de Biologia/CCS, Bloco H, Universidade Federal do Rio de Janeiro, 21941-590 Rio de Janeiro, RJ, Brasil.

Fax: 5521 270-3883; E-mail: vector control @ nppn.ufrj.br

Recebido para publicação em 19/1/98.
} 
As espécies de mosquito Aedes aegypti (Linnaeus, 1762) e Culex quinquefasciatus Say, 1823 são de grande importância médico sanitária, por serem transmissoras da dengue e da filariose, respectivamente.

O melhoramento das condições sanitárias, através da eliminação dos locais de criação, seria o método de controle ideal. No entanto, ainda hoje, o método mais utilizado é o controle químico, aonde são utilizados diversos inseticidas sintéticos como organoclorados, organofosforados, carbamatos e piretróides. Dificuldades no controle desses insetos, têm sido encontradas, em função do surgimento da resistência a vários grupos de inseticidas. Por isto, novas alternativas de controle vêm sendo estudadas. Como exemplo, temos o controle biológico através de peixes ${ }^{1}$, moluscos, crustáceos, insetos, protozoários, fungos, nematódeos, mas principalmente através de bactérias 210 , os análogos de hormônio juvenil que interferem na pupação e na emergência de adultos 11 e o uso de poliestireno expandido, que vem sendo empregado, na forma de partículas flutuantes, em ambientes confinados, impedindo a postura 16 .

Propriedades inseticidas de produtos químicos de origem vegetal também vêm sendo testadas contra insetos e ácaros 4 . Em uma revisão feita por Heal et al7, 2.500 espécies de plantas foram examinadas quanto às suas propriedades inseticidas. Inicialmente, extratos aquosos das plantas foram injetados em Periplaneta americana e aplicados topicamente nas espécies Blatella germanica e Oncopeltus fasciatus. Como exemplo, o extrato aquoso de Agave americana foi injetado em adultos de Periplaneta americana, demonstrando uma capacidade de paralisar $100 \%$ dos indivíduos dois dias após tratamento com o extrato obtido de $0,4 \mathrm{mg}$ de planta por $100 \mathrm{mg}$ de peso do inseto. No entanto, quando aplicado topicamente em Oncopeltus fasciatus e Blatella germanica, o mesmo extrato não demonstrou toxicidade. Por este motivo, Agave americana não foi submetida aos ensaios biológicos posteriores, o mesmo acontecendo com outras 1.800 das 2.500 espécies de plantas estudadas. Com as 700 espécies restantes, foram feitas extrações de várias partes das plantas (raiz, caule, sementes, etc.), utilizando 3 tipos de solventes (álcool, éter de petróleo e água com clorofórmio). A partir dos extratos obtidos, soluções foram preparadas e submetidas a ensaios biológicos com seis espécies de insetos: Blatella germanica, Oncopeltus fasciatus, Tineola bisselliella,
Attagenus piceus, Aedes aegypti, Anopheles quadrimaculatus e Tribolium confusum. Para Aedes aegypti, utilizaram-se larvas com 3 dias de idade que foram submetidas a concentrações de 20, 50 e 200ppm, procurando-se a concentração do extrato que acarretaria em aproximadamente $50 \%$ de mortalidade. $\mathrm{Na}$ concentração de 20ppm, 16 espécies de plantas pertencentes às famílias Annonaceae, Araceae, Asteraceae, Chenopodiaceae, Flacourtiaceae, Leguminoseae e Liliaceae tiveram atividade inseticida, com 50ppm, 17 espécies das famílias Asteraceae, Convolvulaceae, Leguminoseae, Liliaceae e Simaroubaceae mostraram atividade e com 200ppm, 150 plantas foram ativas. As demais espécies de plantas ocasionaram menos de $50 \%$ de mortalidade na concentração de 200ppm ou não tiveram atividade larvicida.

Apesar de neste trabalho não se ter demonstrado atividade inseticida para o gênero Agave, mais recentemente foi feito um estudo em que o extrato de Agave americana apresentou atividade larvicida em Aedes fluviatilis na concentração de $100 \mathrm{ppm}$ de extrato bruto, causando $93 \%$ de mortalidade em 48 horas 5 . Em um outro estudo observou-se a atividade larvicida de Agave americana, A. americana marginata e $A$. arrovirence em larvas de primeiro e quarto estádios de Anopheles stephensi, Aedes aegypti e Culex quinquefasciatus 6 . Os extratos brutos foram diluídos em água em concentrações que correspondiam ao intervalo de 1.250 até $5.000 \mathrm{ppm}$ e as larvas foram expostas por 24 horas. Os resultados indicaram que as três espécies de plantas possuem atividade larvicida nestas altas concentrações, sendo que a $A$. americana mostrou ser a mais eficiente, registrando mortalidade acima de $50 \%$ na concentração de 1250ppm.

No Brasil, uma planta deste gênero de grande interesse econômico é a Agave sisalana. Esta planta é cultivada em larga escala no Nordeste brasileiro, e, devido à sua perfeita adaptação ao clima semi-árido e resistência à seca, acabou se transformando na principal cultura de várias áreas da região. Na Bahia, o sisal representa o segundo produto na pauta de exportação agrícola13, estimando-se que aproximadamente 150 mil famílias vivam às custas desta indústria.

$\mathrm{Da}$ folha da $A$. sisalana aproveitam-se apenas as fibras, que são usadas na fabricação de cordas, tapetes, etc., sendo a parte aquosa e o bagaço considerados resíduos. Estes, no 
entanto, poderiam ser utilizados como matéria prima abundante e barata para outras aplicações, já que as fibras representam somente $4 \%$ do seu peso, e os resíduos sólidos e aquosos os restantes $96 \%$. Nas tentativas anteriores de aproveitamento deste resíduo ele foi testado como biofertilizante ou fornecido diretamente ao gado bovino como alimento na época das secas mas sem resultado expressivo em grande escala13.

Nestes rejeitos encontram-se saponinas esteroidais 14 , também presentes em diversas outras plantas e animais marinhos. As saponinas possuem atividade hemolítica e são tóxicas para peixes. Atividades biológicas e farmacológicas como antiinflamatória, antialérgica e imunomoduladora entre outras, foram também citadas na literatura 8 . No caso da $A$. sisalana, as sapogeninas (porção não glicosídica de uma saponina) encontradas são: a tigogenina, a diosgenina e a hecogenina, registrando-se um maior percentual para esta última. Vários trabalhos quantificam as sapogeninas na planta do sisal. Em um deles obteve-se 0,67g por litro de suco $^{9}$ e em outro um pouco menos:
$54,54 \mathrm{mg} / 100 \mathrm{ml}$. Para utilização industrial, existem algumas plantas que se prestam à obtenção de esteróides (como por exemplo as espécies de Dioscorea), mas a hecogenina obtida da $A$. sisalana possui uma molécula saturada onde se faz necessária a inclusão de etapas adicionais na cadeia de transformação que leva aos hormônios esteroidais tornando-a menos interessante para a indústria.

Os objetivos deste trabalho são: estudar as possibilidades de uso do resíduo líquido de $A$. sisalana resultante do processo de extração das fibras na indústria sisaleira, como larvicida para controle de Aedes aegypti e Culex quinquefasciatus; desenvolver formulações do extrato de $A$. sisalana através do suco desidratado, visando obter um produto seco, evitando a fermentação e consequente perda do produto e aumentando a concentração dos possíveis princípios ativos para facilitar o transporte e a manipulação e, finalmente, determinar os princípios ativos dos extratos e as doses a serem usadas no campo para o controle desses insetos.

\section{MATERIAL E MÉTODOS}

As folhas de $A$. sisalana, provenientes do município de Valente, BA, região de clima semiárido com temperatura média anual de $23,8^{\circ} \mathrm{C}$, após cortadas, foram picadas e trituradas. Foi feita uma filtragem em tecido de algodão a fim de separar o extrato aquoso das fibras e em seguida, uma desidratação em estufa a $40^{\circ} \mathrm{C}$ produzindo o extrato bruto desidratado (EBDA), posteriormente reduzido a pó em um graal.

A extração das saponinas do EBDA foi realizada em aparelho de Soxhlet, utilizando-se $20 \mathrm{~g}$ de extrato e $500 \mathrm{ml}$ de metanol, durante 24 horas. O extrato metanólico foi concentrado em evaporador rotatório, sob pressão reduzida $(30 \mathrm{~mm} \mathrm{Hg})$, a um volume de $100 \mathrm{ml}$ e submetido a precipitação com 4 volumes de acetato de etila. Após 24 horas, a água-mãe foi decantada, o precipitado dissolvido em $5 \mathrm{ml}$ de água e submetido a liofilização. À água-mãe restante $(500 \mathrm{ml})$ foi adicionado novamente acetato de etila $(400 \mathrm{ml})$ a fim de precipitar também as saponinas com pesos moleculares menores. Após 24 horas, a água-mãe $(900 \mathrm{ml})$ foi decantada, o precipitado obtido foi dissolvido em $10 \mathrm{ml}$ de água e submetido a liofilização3.
Nos ensaios biológicos da ação larvicida do EBDA foram utilizadas larvas de 30 estádio de $A$. aegypti e C. quinquefasciatus, das colônias do laboratório de biologia do NPPN-UFRJ, oriundas do município do Rio de Janeiro e mantidas a $27 \pm 2^{\circ} \mathrm{C}$. Grupos de 25 larvas foram expostos a diferentes concentrações de EBDA e as percentagens de mortalidade determinadas após 24 horas de contato. Cada ensaio foi repetido pelo menos 4 vezes. Em seguida novos ensaios foram programados com concentrações mais próximas entre si, visando-se obter pelo menos três delas que induzissem à mortalidade entre 10 e $90 \%$, para permitir a determinação da linha básica de suscetibilidade. Os dados foram plotados em gráficos e com isto foi interpolada a CL50 e extrapoladas as CL90 e 95, segundo a OMS15. Esta mesma metodologia também foi utilizada nos demais ensaios biológicos variando apenas o tempo de exposição das larvas ao EBDA (96 horas). Para a análise dos dados foi utilizada a Análise de Probitos do pacote estatístico SPSS (Statistical Product and Service Solution for Windows). 


\section{RESULTADOS}

De $4,82 \mathrm{~kg}$ de folhas de Agave sisalana foram obtidos $1.380 \mathrm{ml}$ de extrato bruto, que, após desidratado, resultou em 107,2g de extrato seco apresentando coloração marrom.

As concentrações letais obtidas para A. aegypti foram CL50 322ppm, CL90 980ppm e CL95 1343ppm e para C. quinquefasciatus CL50 183ppm, CL90 408ppm e CL95 512ppm (Tabela 1).

A CL50 para as larvas de $A$. aegypti é 1,79 vezes maior que a CL50 para $C$. quinquefasciatus, sendo assim, seria preciso uma concentração mais elevada para eliminar as larvas de $A$. aegypti. Como esta espécie se desenvolve em água limpa e potável, este fator poderia dificultar o seu uso, pois, para se usar um produto em água potável ele deve ser palatável, não deve provocar coloração forte quando solubilizado em água e não pode ser tóxico na concentração utilizada. Isto não inviabiliza o EBDA para o controle de $A$. aegypti, mas requer um estudo mais detalhado tanto na parte dos ensaios biológicos

Tabela 1 - Suscetibilidade de larvas de $3^{\circ}$ estádio de A. aegypti e C. quinquefasciatus ao EBDA, após exposição de 24 horas.

\begin{tabular}{lccc}
\hline Espécies & $\begin{array}{c}\text { CL50 } \\
(\mathrm{ppm})\end{array}$ & $\begin{array}{c}\text { CL90 } \\
(\mathrm{ppm})\end{array}$ & $\begin{array}{c}\text { CL95 } \\
(\mathrm{ppm})\end{array}$ \\
\hline A. aegypti & 322 & 980 & 1343 \\
C. quinquefasciatus & 183 & 408 & 512 \\
\hline
\end{tabular}

quanto toxicológicos. Para C. quinquefasciatus que se desenvolve em água suja, geralmente de esgotos, os ensaios de toxicidade seriam desejáveis, mas a utilização a nível regional seria aceitável mesmo sem o conhecimento pormenorizado de sua toxicidade. Diante destes resultados o presente trabalho foi direcionado para a atividade do EBDA em larvas de C. quinquefasciatus.

As saponinas foram isoladas e submetidas a ensaios biológicos junto com as substâncias resultantes deste processo de extração. De $20 \mathrm{~g}$ de EBDA, foram obtidos $3,5 \mathrm{~g}$ da fração saponinas de alto peso molecular (apm), 109mg da fração saponinas de baixo peso molecular (bpm) e 780mg da fração água-mãe. No ensaio biológico realizado com estas substâncias, foi utilizada uma concentração de 204ppm, pouco acima da CL50 do EBDA estimada pela Análise de Probitos. Constatamos que a fração de saponinas apm acarretou $50 \%$ de mortalidade, as saponinas bpm $20 \%$, a água-mãe $36 \%$ e o resíduo que foi retirado do cartucho causou 30\% de mortalidade (Tabela 2).

Tabela 2 - Atividade das frações saponinas de alto e baixo pesos moleculares, água-mãe e do resíduo do cartucho, em larvas de terceiro estádio de Culex quinquefasciatus submetidos à 24 horas de contato.

\begin{tabular}{lccc}
\hline Produto & Conc. $(\mathrm{ppm})$ & № de larvas testadas & Mortalidade (\%) \\
\hline saponinas apm & 204 & 50 & 50 \\
saponinas bpm & 204 & 50 & 20 \\
água-mãe & 204 & 50 & 36 \\
resíduo cartucho & 204 & 100 & 30 \\
\hline
\end{tabular}

Todos os produtos apresentaram atividade, mas nenhum se destacou claramente. Aquele que seria o princípio ativo deveria apresentar um percentual de mortalidade maior que o do EBDA e os demais um percentual menos significante. Como isto não ocorreu, podemos deduzir que a atividade deve estar em vários componentes do EBDA.

No ensaio seguinte tratou-se de aumentar o tempo de exposição das larvas de terceiro estádio de $C$. quinquefasciatus às frações de saponinas apm, saponinas bpm, água-mãe e do resíduo 
de EBDA retirado do cartucho, visando reduzir a concentração necessária para uso no campo. A fração saponinas apm manteve o mesmo resultado do ensaio anterior ( $50 \%$ de mortalidade) com 24 horas de exposição, mas em 48 horas atingiu $100 \%$ de mortalidade, demonstrando que podese diminuir a concentração aumentando o tempo de exposição. A fração de saponinas bpm causou $64 \%$ de mortalidade após 96 horas. A fração água-mãe e o resíduo do cartucho atingiram 100\% de mortalidade em 72 horas (Figura 1). Estes resultados confirmam a dedução anterior de que são vários componentes do EBDA que apresentam atividade em larvas de terceiro estádio de Culex quinquefasciatus e também indicam que a concentração necessária diminui aumentando-se o tempo de exposição ao produto.

Diante deste resultado, um outro ensaio foi realizado com o objetivo de reduzir a concentração que seria utilizada em campo. Observou-se o percentual de mortalidade de larvas de terceiro estádio de $C$. quinquefasciatus expostas a concentrações menores do EBDA aumentandose, em contrapartida, o tempo de exposição. Observamos que 150ppm causam 100\% de mortalidade das larvas entre 24 e 48 horas de exposição, que 100 ppm levam a $100 \%$ de mortalidade em 96 horas e que 50ppm não acarreta $100 \%$ de mortalidade em até 96 horas de contato, momento em que o ensaio foi interrompido (Figura 2). Isto demonstra a possibilidade de se reduzir a concentração a ser utilizada em campo para 100ppm, desde que se propicie aumento do tempo de exposição, fazendo do EBDA um produto viável para o controle de larvas de $C$. quinquefasciatus. $\mathrm{Na}$ eventualidade de se necessitar de um controle mais rápido, pode-se utilizar a concentração de $150 \mathrm{ppm}$ que elimina as larvas no período

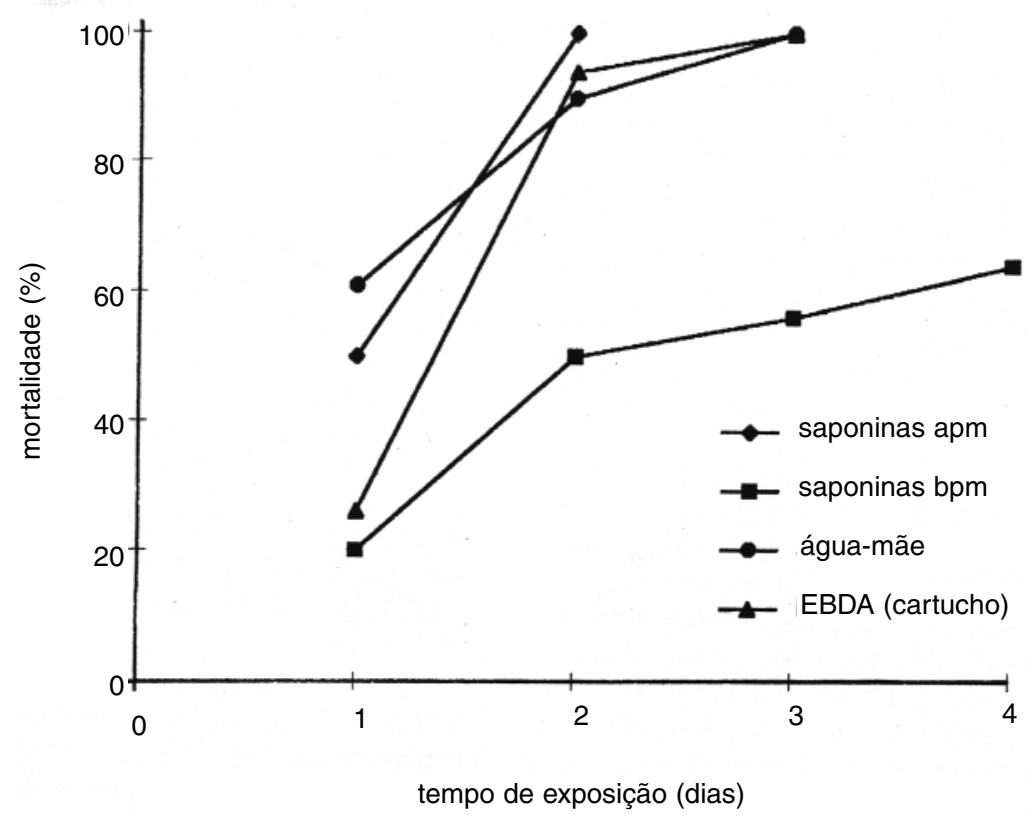

Figura 1 - Percentagens de mortalidade de larvas de $3^{\circ}$ estádio de Culex quinquefasciatus submetidas à áqua tratada $\mathrm{com}$ as frações resultantes do processo de extração das saponinas do extrato bruto desidratado de Agave sisalana (EBDA) na concentração de 204ppm, durante 4 dias. 


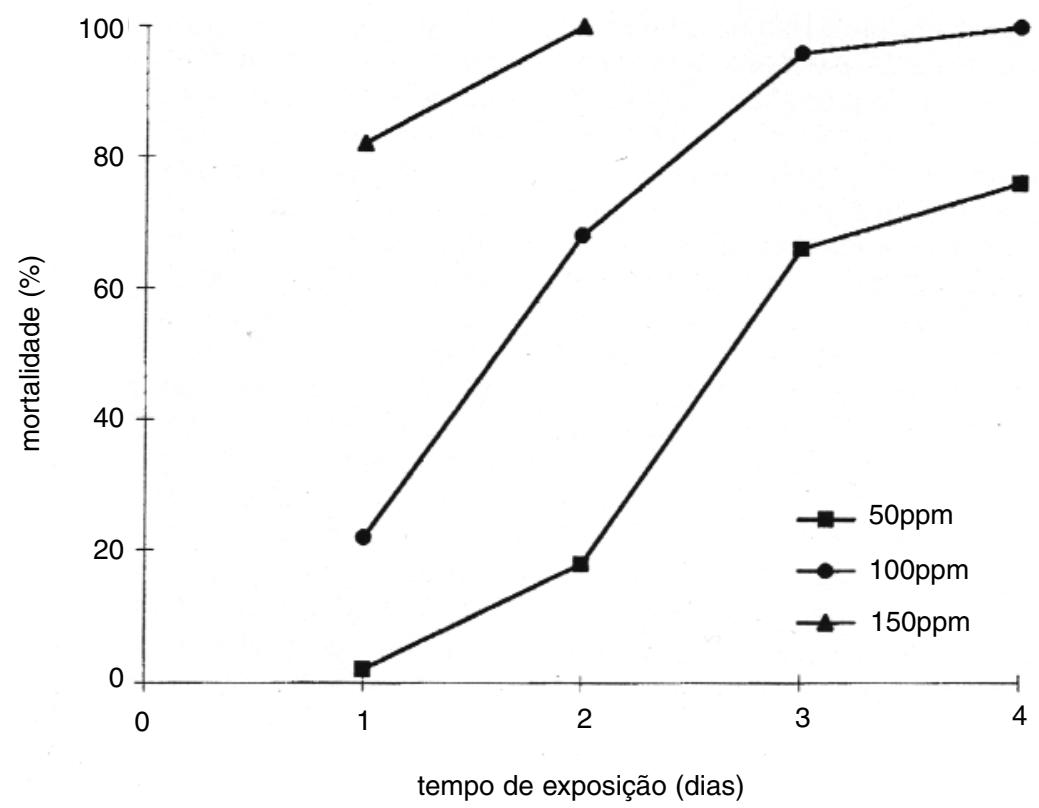

Figura 2 - Percentagens de mortalidade de larvas de 3 e estádio de Culex quinquefasciatus submetidas à áqua tratada com extrato bruto desidratado de Agave sisalana (EBDA) na concentração de 50, 100 e 150ppm, durante 4 dias.

compreendido entre 24 e 48 horas.

\section{DISCUSSÃO}

Em vista das dificuldades encontradas com o uso de inseticidas químicos sintéticos, os produtos de origem natural vêm sendo estudados como alternativa menos tóxica para o meio ambiente e provavelmente de menor custo. De 2.500 plantas estudadas por Heal et al7, 180 mostraram atividade larvicida para $A$. aegypti 7 . Algumas espécies do gênero Agave foram estudadas mais recentemente como larvicidas, e a $A$. americana apresentou atividade para Aedes fluviatilis na concentração de $100 \mathrm{ppm}^{5} \mathrm{e}$ para Anopheles stephensi, Aedes aegypti e Culex quinquefasciatus, em concentrações de extrato bruto muito maiores (1.250-5.000ppm)6. Provavelmente, por terem trabalhado com concentracões tão elevadas não se fez menção da possibilidade de utilização destes extratos em condições de campo. Os trabalhos que se referem às espécies do gênero Agave, não desenvolveram nenhum estudo químico para analisar os possíveis princípios ativos e nenhum se refere a Agave sisalana, a espécie estudada no presente trabalho.
Neste estudo, a espécie $A$. sisalana apresentou atividade larvicida para $C$. quinquefasciatus, e existem fatores importantes que merecem destaque. Considerando que a espécie de mosquito em questão se desenvolve em água suja e estagnada e que nestas condições o produto ali permanece por mais tempo, a concentração de EBDA poderia ser reduzida a $100 \mathrm{ppm}$ pois facilmente se conseguiria um período de exposição de 3 a 4 dias. Isto significa que a quantidade de EBDA utilizada, por exemplo, em 1000 litros de água, seria de $100 \mathrm{~g}$, ou seja, a mesma quantidade utilizada pela Fundação Nacional de Saúde (FNS) para o produto Abate $1 \mathrm{G}$, utilizado para o controle de larvas nas campanhas contra dengue. Isto mostra que é possível a utilização do EBDA como alternativa prática ao produto utilizado pela FNS.

O fato de EBDA ser ativo através da interação de vários dos seus componentes representa também, para indústria sisaleira, uma economia num futuro processo de produção, já que não é necessário nenhuma separação química. 
Além de controlar um mosquito que produz muito incômodo e que tem também importância sanitária em algumas áreas do país, este larvicida irá contribuir para a melhoria sócioeconômica da região nordestina, onde uma população carente e de baixa renda, foi capaz de desenvolver uma indústria de subsistência na qual toneladas de resíduo são desperdiçadas anualmente. Este resíduo obtido a custo zero, poderia ser aproveitado como matéria prima na produção deste larvicida. O processo de fabricação deste larvicida se resumiria em poucas etapas. Poderia ser iniciado por uma prensagem, com a função de separar o restante das fibras e resíduos sólidos da parte aquosa, em seguida uma filtragem, para que sejam retirados todos os demais resíduos que porventura tenham permanecido após a prensagem. Depois o líquido passaria por um processo de desidratação para o qual poder-se-ia aproveitar até mesmo a energia solar abundante na região. Este trabalho oferece portanto uma alternativa de uso de um produto natural, com matéria prima abundante e de baixo custo para o controle de Culex quinquefasciatus. Traz também a perspectiva de ocupação de mão-de-obra local, de agregação de valor a um produto considerado lixo, podendo se constituir numa significativa contribuição para a melhoria

das condições sócio-econômicas da região nordeste do Brasil.

\section{AGRADECIMENTOS}

Os autores agradecem a Elza L.B. Lustosa, Aline T.M. Oliveira, Celso E. Santos, Elizabete G. Costa, Joaquim J. Souza, Marli T.V. Melo e Orbino C. Damião (Laboratório de Biologia -
NPPN/UFRJ) pela condução dos ensaios biológicos e revisão do texto. A Robson $R$. Bernardo (Laboratório de Química de Carboidratos - NPPN/UFRJ) pelo auxílio nas extrações químicas, ao Professor Luis O. Azevedo (Fiocruz, RJ), pelo apoio na análise estatística

deste trabalho, e ainda ao Professor Miguel de Simoni (COPPE/UFRJ) pelo apoio constante.

\section{REFERÊNCIAS BIBLIOGRÁFICAS}

1. Alio AY, Isaq A, Delfini LF. Field trial on the impact of Oreochromis opilurus spilurus on malaria transmission in Northern Somalia. WHO/VBC/8,910, 1985.

2. Alves SB, Andrade CFS, Capalbo OMF. Controle microbiano de insetos. Ed. Manole, São Paulo, 1986.

3. Bernardo RR, Pinto AV, Parente JP. Steroidal saponins from Smilax officinalis. Phytochemistry 43:465-469, 1996.

4. Brum APO, Estudo para uma alternativa carrapaticida de controle do Boophilus microplus em bovinos. Tese de Mestrado, Universidade Federal do Rio de Janeiro, Rio de Janeiro, RJ, Brasil, 1993.

5. Consoli RAGB, Mendes NM, Pereira JP, Santos BS, Lamounier MLA. Influência de diversos derivados de vegetais na sobrevida das larvas de Aedes fluviatilis (Lutz) (Diptera: Culicidae) em laboratório. Memórias do Instituto Oswaldo Cruz 83:87-93, 1988.

6. Daharam Shaktu NS, Menon PKM. Larvicidal Property of Three species of genus Agave (Fam: Amaryllidaceae). Journal of Communication Disorders 15:135-137, 1983.

7. Heal RE, Rogers EF, Wallace RT, Starnes O. A survey of plants for insecticidal activity, Lloydia 13:89-162, 1950.

8. Lacaille-Dubois MA, Wagner HA. Review of the biological and pharmacological activities of saponins. Phytomedicine 4:363-386, 1996.
9. Mors WB, Sharaphin N. A obtenção de esteróides do sisal. Revista Brasileira de Tecnologia 4:153-165, 1973.

10. Oliveira Filho AM, Costa EG, Melo MTV, Santos CE. Activity and persistence of Bacillus thuringiensis Pyriproxifen and temefós against Culex quinquefasciatus and Aedes aegypti larvae. Revista da Sociedade Brasileira de Medicina Tropical 29 (supl I):62, 1996.

11. Oliveira Filho AM, Melo MTV, Silva EL, Santos CE, Costa $E G$, Yasuda JA. Juvenile hormone analogue against Culex quinquefasciatus and Aedes aegypti. In: Abstract of $1^{\text {st }}$ International Congress of Parasitology and Tropical Medicine, Kuala Lumpur, Malásia, p. 145, 1994.

12. Perez N, Matteo C, Cuervo AC, Marcos MN, Blunden G. Determinacion cuantitativa de hecogenina y tigogenina del sisal venezuelano utilizando cromatografia líquida de alta resolucion. Revista Latinoamericana Química 2:102103, 1989.

13. Silva, ALV, Oliveira IF, Costa IS, Estrela L. APAEB: uma história de fibra, luta e subsistência. Editora Feira de Santana, BA, Brasil, 1993.

14. Wilkomirski B, Bobeyko VA, Kintia PK. New steroidal saponins of Agave americana. Phytochemistry 14:26572659, 1975.

15. World Health Organization. Insecticide resistance and vector control. World Health Organization Technical Reports Series. no 443, 1970.

16. World Health Organization. Control of limphatic filariasis. 